\title{
A General Class of Tests for Testing Homogeneity of Location Parameters against Umbrella Alternatives
}

\author{
Manish Goyal", Narinder Kumar* \\ Department of Statistics \\ Panjab University, Chandigarh, India \\ E-mails: \#manishgoyal33@gmail.com, "nkumar@pu.ac.in \\ ${ }^{*}$ Corresponding author
}

(Received January 26, 2018; Accepted March 17, 2018)

\begin{abstract}
In this paper, a general class of non-parametric tests for testing homogeneity of location parameter against umbrella alternatives is proposed. Testing for umbrella alternatives has many applications in the field of biology, medicine, botany, dose level testing, engineering, economics, psychology, zoology. As an example, the effectiveness of a drug is likely to increase with increase of dose up to a certain level and then its effect begins to decrease. The proposed test is based on linear combination of two-sample $U$-statistics. The null distribution of the test statistics is developed. We compare the test with some other competing tests in terms of Pitman asymptotic relative efficiency. To see execution of the test, a numerical example is provided. Simulation study is carried out to assess the power of proposed class of tests.
\end{abstract}

Keywords- Non-parametric tests, Umbrella alternatives, Null distribution, Pitman efficiency, Simulation study.

\section{Introduction}

Suppose there are $k(k \geq 2)$ independent random samples $X_{l 1}, \ldots, X_{l n_{l}} ; l=1, \ldots, k$, of size $n_{l}$ with absolute continuous cumulative distribution function (cdf) of $l^{\text {th }}$ sample as $F_{l}(x)=$ $F\left(x-\theta_{l}\right), l=1, \ldots, k$. Now it is of interest to check whether all the samples are from a common distribution or there is an umbrella pattern in their location parameter $\theta_{l}{ }^{\prime} s$. Thus we wish to test the null hypothesis:

$$
H_{0}: \theta_{1}=\ldots=\theta_{k}
$$

against the umbrella alternative

$$
H_{1}: \theta_{1} \leq \ldots \leq \theta_{p-1} \leq \theta_{p} \geq \theta_{p+1} \geq \ldots \geq \theta_{k}
$$

with at least one strict inequality and $p$ is the known peak of umbrella alternative.

For $p=k$ or $p=1$, the umbrella alternative corresponds to ordered alternative. For testing of ordered alternative, Jonckheere (1954) and Terpstra (1952) firstly proposed a test which is further modified by a number of researchers and references may be made to Amita and Kochar (1989), Kumar et al. (1994 a, b), Büning and Kössler (1996), Kumar et al. (2003), Kössler (2005), Goyal and Kumar (2017) and Kumar and Goyal (2018).

The umbrella alternatives firstly introduced by Archambault et al. (1977). For testing of umbrella alternative, Mack and Wolfe (1981) firstly proposed a test which is further modified by a number 
International Journal of Mathematical, Engineering and Management Sciences

Vol. 3, No. 4, 498-512, 2018

https://dx.doi.org/10.33889/IJMEMS.2018.3.4-036

of researchers and references may be made to Simpson and Margolin (1986), Büning and Kössler (1997), Kössler (2006), Patil (2007), Bhat (2009) and Gökpinar and Gökpinar (2016).

For two-sample location problem, Kumar (2015) proposed a general class of distribution-free test, which is a generalization of two-sample tests proposed by Wilcoxon-Mann-Whitney (1945, 1947), Deshpande and Kochar (1982), Kumar (1997) and Kumar et al. (2003). In this paper, we proposed a general class of distribution-free tests for $k$-sample location problem with umbrella alternative based on linear combination of two-sample test statistics proposed by Kumar (2015).

The test statistics is introduced in Section 2. The asymptotic distribution of the test statistics is derived in Section 3. Optimal choice of weights, in order to attain maximum efficiency of the proposed test is discussed in Section 4. We compare the proposed class of tests with respect to some other existing tests in Section 5. For illustrative purpose, a real life data example is discussed in Section 6. Simulation study is carried out in Section 7 to assess the power of the proposed class of tests.

\section{Proposed Test Statistics}

Firstly we consider the two-sample $U$-statistics proposed by Kumar (2015). Suppose $c$ and $d$ be fixed sub-sample sizes such that $1 \leq c, d \leq \min \left(n_{1}, \ldots, n_{k}\right)$. Let $i$ and $j$ be fixed integers such that $1 \leq i \leq c$ and $1 \leq j \leq d$. Now, for $l<m ; l, m=1, \ldots, k$, define the following kernel:

$$
h_{i, j}\left(X_{l 1}, \ldots, X_{l c} ; X_{m 1}, \ldots, X_{m d}\right)= \begin{cases}2 & \text { if } X_{i: c}^{(l)}<X_{j: d}^{(m)} \text { and } X_{c-i+1: c}^{(l)}<X_{d-j+1: d}^{(m)} \\ 1 & \text { if } X_{i: c}^{(l)}<X_{j: d}^{(m)} \text { or } X_{c-i+1: c}^{(l)}<X_{d-j+1: d}^{(m)} \\ 0 & \text { otherwise }\end{cases}
$$

where $X_{i: c}^{(l)}\left(X_{c-i+1: c}^{(l)}\right)$ is the $i^{\text {th }}\left((c-i+1)^{t h}\right)$ order statistics in a sub-sample of size $c$ from the $l^{\text {th }}$ sample and likewise $X_{j: d}^{(m)}\left(X_{d-j+1: d}^{(m)}\right)$ is the $j^{\text {th }}\left((d-j+1)^{t h}\right)$ order statistics in a subsample of size $d$ from the $m^{\text {th }}$ sample.

The Kumar (2015) two-sample $U$-statistics associated with kernel $h_{i, j}$ is:

$$
U_{c, d ; i, j}^{(l, m)}=\left[\left(\begin{array}{c}
n_{l} \\
c
\end{array}\right)\left(\begin{array}{c}
n_{m} \\
d
\end{array}\right)\right]^{-1} \sum h_{i, j}\left(X_{l \alpha_{1}}, \ldots, X_{l \alpha_{c}} ; X_{m \beta_{1}}, \ldots, X_{m \beta_{d}}\right),
$$

where $\Sigma$ donates the sum over all possible combinations of $c$ integers $\left(\alpha_{1}, \ldots, \alpha_{c}\right)$ chosen from $\left(1, \ldots, n_{l}\right)$ and all possible combinations of $d$ integers $\left(\beta_{1}, \ldots, \beta_{d}\right)$ chosen from $\left(1, \ldots, n_{m}\right)$.

Now, we propose a class of test statistics $T_{c, d ; i, j}$ for testing $H_{0}$ against $H_{1}$ as:

$$
T_{c, d ; i, j}=T_{c, d ; i, j}^{(1)}+T_{c, d ; i, j}^{(2)}
$$

where $T_{c, d ; i, j}^{(1)}$ and $T_{c, d ; i, j}^{(2)}$ are test statistics based on linear combination of $U_{c, d ; i, j}^{(l, m)}$ and defined as: 
International Journal of Mathematical, Engineering and Management Sciences

Vol. 3, No. 4, 498-512, 2018

https://dx.doi.org/10.33889/IJMEMS.2018.3.4-036

$$
T_{c, d ; i, j}^{(1)}=\sum_{l=1}^{p-1} a_{l} U_{c, d ; i, j}^{(l, l+1)} \text { and } T_{c, d ; i, j}^{(2)}=\sum_{l=p}^{k-1} a_{l} U_{c, d ; i, j}^{(l+1, l)}
$$

where $a_{l}, l=1, \ldots, k-1$ are suitably chosen real constants. Test is to reject $H_{0}$ for large values of $T_{c, d ; i, j}$.

In particular,

For $c=d=1 ; i=j=1, T_{c, d ; i, j}$ corresponds to test statistics proposed by Bhat (2009).

For $i=(c+1) / 2, j=(d+1) / 2$, with $c, d$ are odd positive integers, then $T_{c, d ; i, j}$ corresponds to test statistics proposed by Patil (2007, Chapter 8).

By putting $a_{l}=1, l=1, \ldots, k-1$, we get Mack-Wolfe form of the proposed test statistics as:

$$
V_{c, d ; i, j}=\sum_{l=1}^{p-1} U_{c, d ; i, j}^{(l, l+1)}+\sum_{l=p}^{k-1} U_{c, d ; i, j}^{(l+1, l)}
$$

\section{Distribution of the Proposed Test Statistics}

The expectation of $U_{c, d ; i, j}^{(l, l+1)}$ is:

$$
\begin{aligned}
E\left[U_{c, d ; i, j}^{(l, l+1)}\right]=\left[\left(\begin{array}{c}
n_{l} \\
c
\end{array}\right)\left(\begin{array}{c}
n_{l+1} \\
d
\end{array}\right)\right]^{-1} \sum E\left[h_{i, j}\left(X_{l \alpha_{1}}, \ldots, X_{l \alpha_{c}} ; X_{(l+1) \beta_{1}}, \ldots, X_{(l+1) \beta_{d}}\right)\right] \\
=E\left[h_{i, j}\left(X_{l \alpha_{1}}, \ldots, X_{l \alpha_{c}} ; X_{(l+1) \beta_{1}}, \ldots, X_{(l+1) \beta_{d}}\right)\right] \\
=P\left(X_{i: c}^{(l)}<X_{j: d}^{(l+1)}\right)+P\left(X_{c-i+1: c}^{(l)}<X_{d-j+1: d}^{(l+1)}\right) \\
=\int_{-\infty}^{\infty} F_{i: c}^{(l)}(y) d\left[F_{j: d}^{(l+1)}(y)\right]+\int_{-\infty}^{\infty} F_{c-i+1: c}^{(l)}(y) d\left[F_{d-j+1: d}^{(l+1)}(y)\right],
\end{aligned}
$$

where $F_{i: c}^{(l)}(y)\left[F_{j: d}^{(l+1)}(y)\right]$ is the cdf of $X_{i: c}^{(l)}\left[X_{j: d}^{(l+1)}\right]$ and $F_{c-i+1: c}^{(l)}(y)\left[F_{d-j+1: d}^{(l+1)}(y)\right]$ is the cdf of $X_{c-i+1: c}^{(l)}\left[X_{d-j+1: d}^{(l+1)}\right]$. Here $d[]$ stands for the derivative of the expression inside the brackets.

Under $H_{0}$,

$$
E\left[U_{c, d ; i, j}^{(l, l+1)}\right]=1, \text { for all } c, d ; i, j
$$

Similarly, under $H_{0}$,

$$
E\left[U_{c, d ; i, j}^{(l+1, l)}\right]=1, \text { for all } c, d ; i, j
$$


International Journal of Mathematical, Engineering and Management Sciences

Vol. 3, No. 4, 498-512, 2018

https://dx.doi.org/10.33889/IJMEMS.2018.3.4-036

Therefore the expectation of class of test statistics $T_{c, d ; i, j}$ is:

$$
\begin{aligned}
E\left[T_{c, d ; i, j}\right] & =\sum_{l=1}^{p-1} a_{l} E\left[U_{c, d ; i, j}^{(l, l+1)}\right]+\sum_{l=p}^{k-1} a_{l} E\left[U_{c, d ; i, j}^{(l+1, l)}\right] \\
& =\sum_{l=1}^{p-1} a_{l}+\sum_{l=p}^{k-1} a_{l}=\sum_{l=1}^{k-1} a_{l}
\end{aligned}
$$

and the expectation of class of test statistics $V_{c, d ; i, j}$ is:

$$
E\left[V_{c, d ; i, j}\right]=k-1
$$

As $U_{c, d ; i, j}^{(l, m)}$ is two-sample $U$-statistics and the proposed class of test statistics $T_{c, d ; i, j}$ is the linear combination of $U_{c, d ; i, j}^{(l, m)}$, so using the results given by Lehmann (1963) and Puri (1965) and applying the transformation theorem due to Serfling (1980, p.122), we found the asymptotic distribution of class of test statistics $T_{c, d ; i, j}$ as given in the following theorem.

Theorem 1. Let $N=\sum_{l=1}^{k} n_{l}$. The asymptotic distribution of $N^{1 / 2}\left(T_{c, d ; i, j}-E\left[T_{c, d ; i, j}\right]\right)$ as $N \rightarrow$ $\infty$ in such a way that $n_{l} / N \rightarrow \lambda_{l}, 0<\lambda_{l}<1$, for $l=1, \ldots, k$ is normal with mean zero and variance $\zeta_{T}$, where

$$
\begin{aligned}
& \zeta_{T}=\operatorname{Var}\left(T_{c, d ; i, j}\right)=\zeta_{1}+\zeta_{2}+2 \zeta_{12} \\
& \zeta_{1}=\operatorname{Var}\left(T_{c, d ; i, j}^{(1)}\right)=\underline{a}_{1}^{\prime} \sum_{1} \underline{a}_{1}, \underline{a}_{1}=\left(a_{1}, \ldots, a_{p-1}\right) \\
& \zeta_{2}=\operatorname{Var}\left(T_{c, d ; i, j}^{(2)}\right)=\underline{a}_{2}^{\prime} \sum_{2} \underline{a}_{2}, \underline{a}_{2}=\left(a_{p}, \ldots, a_{k-1}\right) \\
& \zeta_{12}=\operatorname{Cov}\left(T_{c, d ; i, j}^{(1)}, T_{c, d ; i, j}^{(2)}\right)=a_{p-1} a_{p} \operatorname{Cov}\left(U_{c, d ; i, j}^{(p-1, p)}, U_{c, d ; i, j}^{(p+1, p)}\right)
\end{aligned}
$$

Now substitute (2), (3) and (4) in (1), we get,

$$
\zeta_{T}=\underline{a}_{1}^{\prime} \sum_{1} \underline{a}_{1}+\underline{a}_{2}^{\prime} \sum_{2} \underline{a}_{2}+2 a_{p-1} a_{p} \operatorname{Cov}\left(U_{c, d ; i, j}^{(p-1, p)}, U_{c, d ; i, j}^{(p+1, p)}\right)
$$

It can be seen that under $H_{0}, \sum_{1}=\left(\left(\sigma_{l, m}^{(1)}\right)\right)$ is given as: 
International Journal of Mathematical, Engineering and Management Sciences

Vol. 3, No. 4, 498-512, 2018

https://dx.doi.org/10.33889/IJMEMS.2018.3.4-036

$$
\left(\left(\sigma_{l, m}^{(1)}\right)\right)= \begin{cases}c^{2} \rho_{c, d ; i, j}\left(\frac{1}{\lambda_{l}}+\frac{1}{\lambda_{l+1}}\right) & \text { for } l=m=1, \ldots, p-1 \\ -c^{2} \rho_{c, d ; i, j}\left(\frac{1}{\lambda_{l+1}}\right) & \text { for } m=l+1 ; l=1, \ldots, p-2 \\ -c^{2} \rho_{c, d ; i, j}\left(\frac{1}{\lambda_{l}}\right) & \text { for } m=l-1 ; l=2, \ldots, p-1 \\ 0 & \text { otherwise }\end{cases}
$$

and $\sum_{2}=\left(\left(\sigma_{l, m}^{(2)}\right)\right)$ is given as:

$$
\left(\left(\sigma_{l, m}^{(2)}\right)\right)= \begin{cases}c^{2} \rho_{c, d ; i, j}\left(\frac{1}{\lambda_{l}}+\frac{1}{\lambda_{l+1}}\right) & \text { for } l=m=p, \ldots, k-1 \\ -c^{2} \rho_{c, d ; i, j}\left(\frac{1}{\lambda_{l+1}}\right) & \text { for } m=l+1 ; l=p, \ldots, k-2 \\ -c^{2} \rho_{c, d ; i, j}\left(\frac{1}{\lambda_{l}}\right) & \text { for } m=l-1 ; l=p+1, \ldots, k-1 \\ 0 & \text { otherwise }\end{cases}
$$

and

$$
\operatorname{Cov}\left(U_{c, d ; i, j}^{(p-1, p)}, U_{c, d ; i, j}^{(p+1, p)}\right)=c^{2} \rho_{c, d ; i, j} / \lambda_{p}
$$

where $\rho_{c, d ; i, j}$ is given by Kumar (2015) as:

$$
\begin{aligned}
\rho_{c, d ; i, j} & \left(\begin{array}{l}
d \\
j
\end{array}\right)^{2} j^{2} a_{c, d ; i, j}^{2}\left[\frac{A_{c, d ; i, j}^{2}}{a_{c, d ; i, j}^{2}}-\frac{2 A_{c, d ; i, j}}{a_{c, d ; i, j}} \sum_{r=i+j-1}^{c+d-1}\left(\begin{array}{c}
c+d-1 \\
r
\end{array}\right) \beta(r+1, c+d-r)\right. \\
& +\sum_{r=i+j-1}^{c+d-1} \sum_{s=i+j-1}^{c+d-1}\left(\begin{array}{c}
c+d-1 \\
r
\end{array}\right)\left(\begin{array}{c}
c+d-1 \\
s
\end{array}\right) \beta(r+s+1,2 c+2 d-r-s-1) \\
& +\sum_{r=c+d-i-j+1}^{c+d-1} \sum_{s=c+d-i-j+1}^{c+d-1}\left(\begin{array}{c}
c+d-1 \\
r
\end{array}\right)\left(\begin{array}{c}
c+d-1 \\
s
\end{array}\right) \beta(r+s+1,2 c+2 d-r-s-1) \\
& +2 \sum_{r=i+j-1}^{c+d-1} \sum_{s=c+d-i-j+1}^{c+d-1}\left(\begin{array}{c}
c+d-1 \\
r
\end{array}\right)\left(\begin{array}{c}
c+d-1 \\
s
\end{array}\right) \beta(r+s+1,2 c+2 d-r-s-1) \\
& \left.-\frac{2 A_{c, d ; i, j}}{a_{c, d ; i, j}} \sum_{r=c+d-i-j+1}^{c+d-1}\left(\begin{array}{c}
c+d-1 \\
r
\end{array}\right) \beta(r+1, c+d-r)\right]-1
\end{aligned}
$$


International Journal of Mathematical, Engineering and Management Sciences

Vol. 3, No. 4, 498-512, 2018

https://dx.doi.org/10.33889/IJMEMS.2018.3.4-036

with

$$
\begin{aligned}
A_{c, d ; i, j}= & \sum_{r=c-i+1}^{c-1}\left(\begin{array}{c}
c-1 \\
r
\end{array}\right) \beta(r+d-j+1, c-r+j-1) \\
& +\sum_{r=i}^{c-1}\left(\begin{array}{c}
c-1 \\
r
\end{array}\right) \beta(r+j, c+d-r-j)+2\left(\begin{array}{c}
c-1 \\
i-1
\end{array}\right) \beta(i+j-1, c+d-i-j+1)
\end{aligned}
$$

and

$a_{c, d ; i, j}=\left(\begin{array}{c}c-1 \\ i-1\end{array}\right)\left(\begin{array}{c}c+d-1 \\ i+j-1\end{array}\right)^{-1}(i+j-1)^{-1}$

In case, all sample sizes are equal, i.e. $\lambda_{l}=1 / k$ for all $l=1, \ldots, k$, then substitute (6), (7) and (8) in (5), we get

$$
\begin{aligned}
\zeta_{T} & =2 k c^{2} \rho_{c, d ; i, j}\left\{\sum_{l=1}^{p-1} a_{l}^{2}-\sum_{l=1}^{p-2} a_{l} a_{l+1}+\sum_{l=p}^{k-1} a_{l}^{2}-\sum_{l=p}^{k-2} a_{l} a_{l+1}+a_{p-1} a_{p}\right\} \\
& =2 k c^{2} \rho_{c, d ; i, j}\left\{\sum_{l=1}^{k-1} a_{l}^{2}-\sum_{l=1}^{k-2} a_{l} a_{l+1}+2 a_{p-1} a_{p}\right\} .
\end{aligned}
$$

Similarly, the asymptotic normality of $N^{1 / 2}\left(V_{c, d ; i, j}-E\left[V_{c, d ; i, j}\right]\right)$ is normal with mean zero and variance $\zeta_{V}=6 k c^{2} \rho_{c, d ; i, j}$.

\section{Optimal Choice of Weights}

Since the test statistics $T_{c, d ; i, j}$ is the weighted linear combination of two-sample $U$-statistics, so in this Section, we consider the problem of obtaining the optimal weights, $a_{l}^{\prime} s$, which results to test statistics $T_{c, d ; i, j}$ has maximum efficiency for the sequence of Pitman type alternatives defined as:

$$
H_{N}: F_{l}(x)=F\left(x-N^{-1 / 2} \theta_{l}\right), l=1, \ldots, k .
$$

Under the sequence of Pitman type alternative, the efficacy of the test statistics $T_{c, d ; i, j}$ is

$e\left(T_{c, d ; i, j}\right)=\frac{\left(\sum_{l=1}^{k-1} a_{l}\right)^{2} v_{c, d ; i, j}^{2}}{\zeta_{T}}$

where $\zeta_{T}$ is defined in (9) and 
International Journal of Mathematical, Engineering and Management Sciences

Vol. 3, No. 4, 498-512, 2018

https://dx.doi.org/10.33889/IJMEMS.2018.3.4-036

$$
\begin{array}{r}
v_{c, d ; i, j}=\left(\begin{array}{l}
c \\
i
\end{array}\right) i\left(\begin{array}{l}
d \\
j
\end{array}\right) j\left[\int_{-\infty}^{\infty}(F(y))^{i+j-2}(1-F(y))^{c+d-i-j} f^{2}(y) d y\right. \\
\left.+\int_{-\infty}^{\infty}(F(y))^{c+d-i-j}(1-F(y))^{i+j-2} f^{2}(y) d y\right] .
\end{array}
$$

For efficiency comparisons, we consider the case of equal sample sizes and equally spaced alternatives of the type

$$
\theta_{l}= \begin{cases}l \theta & \text { for } l=1, \ldots, p-1 \\ (2 p-l) \theta & \text { for } l=p, \ldots, k\end{cases}
$$

Now, we deal with finding the optimal weights by using the results of Rao (1973, p.60). The optimal weights $a_{l}^{\prime} s$, for odd $k$ and $p=(k+1) / 2$ are given as:

$a_{l}^{*}=\left\{\begin{array}{ll}\left(\frac{l}{2 k^{2}}\right)(k(p-l)-(p-1)) & \text { for } l=1, \ldots, p-1 \\ \left(\frac{k-l}{2 k^{2}}\right)(k(l+1-p)-(p-1)) & \text { for } l=p, \ldots, k-1\end{array}\right.$.

Using these optimal weights in (10), the efficacy of the test statistics $T_{c, d ; i, j}$ becomes:

$\underbrace{\operatorname{Sup}}_{\underline{a}} e\left(T_{c, d ; i, j}\right)=\frac{\left(k^{4}+2 k^{2}-3\right) v_{c, d ; i, j}^{2}}{48 k^{2} c^{2} \rho_{c, d ; i, j}}$

where $\rho_{c, d ; i, j}$ and $v_{c, d ; i, j}$ are defined in Theorem 1 and (10), respectively.

Similarly, the efficacy of the test statistics $V_{c, d ; i, j}$ becomes:

$e\left(V_{c, d ; i, j}\right)=\frac{(k-1)^{2} v_{c, d i, j}^{2}}{6 k c^{2} \rho_{c, d ; i, j}}$

\section{Comparison of the Proposed Test}

In this Section, we compare the tests $T_{c, d ; i, j}$ and its Mack-Wolfe form $V_{c, d ; i, j}$ with respect to (w.r.t.) some existing tests in terms of the Pitman asymptotic relative efficiency (ARE). We compare the proposed tests w.r.t. $M W$ (Mack and Wolfe, 1981) test, $P_{a, b}$ (Patil, 2007) test, and $B$ (Bhat, 2009) tests.

The AREs of $T_{c, d ; i, j}$ tests w.r.t. $B$ test is same as that of the AREs of $V_{c, d ; i, j}$ tests w.r.t. $M W$ test for given $(c, d ; i, j)$. So, we only found the AREs of $T_{c, d ; i, j}$ test w.r.t. $M W, P_{a, b}$ and $B$ tests. The other AREs can be found using the product rule of ARE, i.e. if there are three tests $T_{1}, T_{2}$ and $T_{3}$ for the same problem then $\operatorname{ARE}\left(T_{1}, T_{2}\right)=\operatorname{ARE}\left(T_{1}, T_{3}\right) \cdot \operatorname{ARE}\left(T_{3}, T_{2}\right)$. 
International Journal of Mathematical, Engineering and Management Sciences

Vol. 3, No. 4, 498-512, 2018

https://dx.doi.org/10.33889/IJMEMS.2018.3.4-036

Remark 1. After submitting the expression of $\rho_{c, d ; i, j}$ and $v_{c, d ; i, j}$ in the expression of efficacy of $T_{c, d ; i, j}$ and $V_{c, d ; i, j}$ as given in (11) and (12) respectively, it can be seen that the efficacy of $T_{c, d ; i, j}$ and $V_{c, d ; i, j}$ depends upon $(c, d ; i, j)$ but through their sum only i.e. $(c+d ; i+j)$.

Remark 2. The AREs of $T_{c, d ; i, j}$ tests w.r.t. $M W$ test depend upon $k$ while AREs of $T_{c, d ; i, j}$ tests w.r.t. $P_{a, b}($ or $B)$ test are independent of $k$.

The AREs of $T_{c, d ; i, j}$ tests w.r.t. $M W$, some members of $P_{a, b}$, and $B$ tests for different underlying distribution are given in Tables 1-7, for different combinations of $(c+d ; i+j)$.

Table 1. AREs of $T_{c, d ; i, j}$ for U-quadratic distribution w.r.t. different tests

\begin{tabular}{|c|c|c|c|c|c|c|c|c|}
\hline & & \multicolumn{7}{|c|}{ Test } \\
\hline \multirow{3}{*}{$c+d$} & $i+j$ & $\begin{array}{c}M W \\
(k=3)\end{array}$ & $\begin{array}{c}M W \\
(k=5)\end{array}$ & $\begin{array}{c}M W \\
(k=7)\end{array}$ & $\begin{array}{c}P_{1,1} \\
\text { (any } k)\end{array}$ & $\begin{array}{c}P_{1,3} \\
\text { (any } k)\end{array}$ & $\begin{array}{c}P_{3,3} \\
\text { (any } k)\end{array}$ & $\begin{array}{c}B \\
\text { (any } k)\end{array}$ \\
\hline 2 & 2 & 1.0000 & 1.0500 & 1.2381 & 1.0000 & 2.1767 & 3.2643 & 1.0000 \\
\hline \multirow{3}{*}{3} & 2 & 1.0000 & 1.0500 & 1.2381 & 1.0000 & 2.1767 & 3.2643 & 1.0000 \\
\cline { 2 - 9 } & 3 & 1.0000 & 1.0500 & 1.2381 & 1.0000 & 2.1767 & 3.2643 & 1.0000 \\
\hline \multirow{4}{*}{4} & 2 & 1.4615 & 1.5346 & 1.8095 & 1.4615 & 3.1813 & 4.7709 & 1.4615 \\
\cline { 2 - 10 } & 3 & 0.4594 & 0.4824 & 0.5688 & 0.4594 & 1.0000 & 1.4997 & 0.4594 \\
\cline { 2 - 10 } & 4 & 1.4615 & 1.5346 & 1.8095 & 1.4615 & 3.1813 & 4.7709 & 1.4615 \\
\hline \multirow{4}{*}{5} & 2 & 2.1254 & 2.2317 & 2.6315 & 2.1254 & 4.6264 & 6.9381 & 2.1254 \\
\cline { 2 - 10 } & 3 & 0.4594 & 0.4824 & 0.5688 & 0.4594 & 1.0000 & 1.4997 & 0.4594 \\
\cline { 2 - 10 } & 4 & 0.4594 & 0.4824 & 0.5688 & 0.4594 & 1.0000 & 1.4997 & 0.4594 \\
\cline { 2 - 10 } & 5 & 2.1254 & 2.2317 & 2.6315 & 2.1254 & 4.6264 & 6.9381 & 2.1254 \\
\hline \multirow{5}{*}{6} & 2 & 2.9068 & 3.0522 & 3.5989 & 2.9068 & 6.3274 & 9.4889 & 2.9068 \\
\cline { 2 - 9 } & 3 & 0.5935 & 0.6232 & 0.7349 & 0.5935 & 1.2920 & 1.9375 & 0.5935 \\
\cline { 2 - 9 } & 4 & 0.3063 & 0.3217 & 0.3793 & 0.3063 & 0.6668 & 1.0000 & 0.3063 \\
\cline { 2 - 9 } & 5 & 0.5935 & 0.6232 & 0.7349 & 0.5935 & 1.2920 & 1.9375 & 0.5935 \\
\cline { 2 - 9 } & 6 & 2.9068 & 3.0522 & 3.5989 & 2.9068 & 6.3274 & 9.4889 & 2.9068 \\
\hline
\end{tabular}


International Journal of Mathematical, Engineering and Management Sciences

Vol. 3, No. 4, 498-512, 2018

https://dx.doi.org/10.33889/IJMEMS.2018.3.4-036

Table 2. AREs of $T_{c, d ; i, j}$ for Uniform distribution w.r.t. different tests

\begin{tabular}{|c|c|c|c|c|c|c|c|c|}
\hline & & \multicolumn{7}{|c|}{ Test } \\
\hline$c+d$ & $i+j$ & $\begin{array}{c}M W \\
(k=3)\end{array}$ & $\begin{array}{c}M W \\
(k=5)\end{array}$ & $\begin{array}{c}M W \\
(k=7)\end{array}$ & $\begin{array}{c}P_{1,1} \\
(\text { any } k)\end{array}$ & $\begin{array}{c}P_{1,3} \\
(\text { any } k)\end{array}$ & $\begin{array}{c}P_{3,3} \\
(\text { any } k)\end{array}$ & $\begin{array}{c}B \\
(\text { any } k)\end{array}$ \\
\hline 2 & 2 & 1.0000 & 1.0500 & 1.2381 & 1.0000 & 1.4571 & 1.7013 & 1.0000 \\
\hline \multirow{2}{*}{3} & 2 & 1.0000 & 1.0500 & 1.2381 & 1.0000 & 1.4571 & 1.7013 & 1.0000 \\
\hline & 3 & 1.0000 & 1.0500 & 1.2381 & 1.0000 & 1.4571 & 1.7013 & 1.0000 \\
\hline \multirow{3}{*}{4} & 2 & 1.2281 & 1.2895 & 1.5205 & 1.2281 & 1.7895 & 2.0893 & 1.2281 \\
\hline & 3 & 0.6863 & 0.7206 & 0.8497 & 0.6863 & 1.0000 & 1.1676 & 0.6863 \\
\hline & 4 & 1.2281 & 1.2895 & 1.5205 & 1.2281 & 1.7895 & 2.0893 & 1.2281 \\
\hline \multirow{4}{*}{5} & 2 & 1.5217 & 1.5978 & 1.8841 & 1.5217 & 2.2174 & 2.5889 & 1.5217 \\
\hline & 3 & 0.6863 & 0.7206 & 0.8497 & 0.6863 & 1.0000 & 1.1676 & 0.6863 \\
\hline & 4 & 0.6863 & 0.7206 & 0.8497 & 0.6863 & 1.0000 & 1.1676 & 0.6863 \\
\hline & 5 & 1.5217 & 1.5978 & 1.8841 & 1.5217 & 2.2174 & 2.5889 & 1.5217 \\
\hline \multirow{5}{*}{6} & 2 & 1.8406 & 1.9327 & 2.2789 & 1.8406 & 2.6821 & 3.1315 & 1.8406 \\
\hline & 3 & 0.7624 & 0.8005 & 0.9439 & 0.7624 & 1.1109 & 1.2970 & 0.7624 \\
\hline & 4 & 0.5878 & 0.6172 & 0.7277 & 0.5878 & 0.8565 & 1.0000 & 0.5878 \\
\hline & 5 & 0.7624 & 0.8005 & 0.9439 & 0.7624 & 1.1109 & 1.2970 & 0.7624 \\
\hline & 6 & 1.8406 & 1.9327 & 2.2789 & 1.8406 & 2.6821 & 3.1315 & 1.8406 \\
\hline
\end{tabular}

Table 3. AREs of $T_{c, d ; i, j}$ for Normal distribution w.r.t. different tests

\begin{tabular}{|c|c|c|c|c|c|c|c|c|}
\hline & & \multicolumn{7}{|c|}{ Test } \\
\hline$c+d$ & $i+j$ & $\begin{array}{c}M W \\
(k=3)\end{array}$ & $\begin{array}{c}M W \\
(k=5)\end{array}$ & $\begin{array}{c}M W \\
(k=7)\end{array}$ & $\begin{array}{c}P_{1,1} \\
(\text { any } k)\end{array}$ & $\begin{array}{c}P_{1,3} \\
(\text { any } k)\end{array}$ & $\begin{array}{c}P_{3,3} \\
(\text { any } k)\end{array}$ & $\begin{array}{c}B \\
(\text { any } k)\end{array}$ \\
\hline 2 & 2 & 1.0000 & 1.0500 & 1.2381 & 1.0000 & 1.0546 & 1.0942 & 1.0000 \\
\hline \multirow{2}{*}{3} & 2 & 1.0000 & 1.0500 & 1.2381 & 1.0000 & 1.0546 & 1.0942 & 1.0000 \\
\hline & 3 & 1.0000 & 1.0500 & 1.2381 & 1.0000 & 1.0546 & 1.0942 & 1.0000 \\
\hline \multirow{3}{*}{4} & 2 & 1.0220 & 1.0731 & 1.2654 & 1.0220 & 1.0778 & 1.1183 & 1.0220 \\
\hline & 3 & 0.9483 & 0.9957 & 1.1740 & 0.9483 & 1.0000 & 1.0376 & 0.9483 \\
\hline & 4 & 1.0220 & 1.0731 & 1.2654 & 1.0220 & 1.0778 & 1.1183 & 1.0220 \\
\hline \multirow{4}{*}{5} & 2 & 1.0345 & 1.0863 & 1.2809 & 1.0345 & 1.0910 & 1.1320 & 1.0345 \\
\hline & 3 & 0.9483 & 0.9957 & 1.1740 & 0.9483 & 1.0000 & 1.0376 & 0.9483 \\
\hline & 4 & 0.9483 & 0.9957 & 1.1740 & 0.9483 & 1.0000 & 1.0376 & 0.9483 \\
\hline & 5 & 1.0345 & 1.0863 & 1.2809 & 1.0345 & 1.0910 & 1.1320 & 1.0345 \\
\hline \multirow{5}{*}{6} & 2 & 1.0317 & 1.0833 & 1.2774 & 1.0317 & 1.0880 & 1.1289 & 1.0317 \\
\hline & 3 & 0.9698 & 1.0183 & 1.2007 & 0.9698 & 1.0227 & 1.0611 & 0.9698 \\
\hline & 4 & 0.9139 & 0.9596 & 1.1315 & 0.9139 & 0.9638 & 1.0000 & 0.9139 \\
\hline & 5 & 0.9698 & 1.0183 & 1.2007 & 0.9698 & 1.0227 & 1.0611 & 0.9698 \\
\hline & 6 & 1.0317 & 1.0833 & 1.2774 & 1.0317 & 1.0880 & 1.1289 & 1.0317 \\
\hline
\end{tabular}


International Journal of Mathematical, Engineering and Management Sciences

Vol. 3, No. 4, 498-512, 2018

https://dx.doi.org/10.33889/IJMEMS.2018.3.4-036

Table 4. AREs of $T_{c, d ; i, j}$ for Logistic distribution w.r.t. different tests

\begin{tabular}{|c|c|c|c|c|c|c|c|c|}
\hline & & \multicolumn{7}{|c|}{ Test } \\
\hline$c+d$ & $i+j$ & $\begin{array}{c}M W \\
(k=3)\end{array}$ & $\begin{array}{c}M W \\
(k=5)\end{array}$ & $\begin{array}{c}M W \\
(k=7)\end{array}$ & $\begin{array}{c}P_{1,1} \\
(\text { any } k)\end{array}$ & $\begin{array}{c}P_{1,3} \\
(\text { any } k)\end{array}$ & $\begin{array}{c}P_{3,3} \\
(\text { any } k)\end{array}$ & $\begin{array}{c}B \\
(\text { any } k)\end{array}$ \\
\hline 2 & 2 & 1.0000 & 1.0500 & 1.2381 & 1.0000 & 1.0119 & 1.0292 & 1.0000 \\
\hline \multirow{2}{*}{3} & 2 & 0.4167 & 1.0500 & 1.2381 & 1.0000 & 1.0119 & 1.0292 & 1.0000 \\
\hline & 3 & 0.2593 & 1.0500 & 1.2381 & 1.0000 & 1.0119 & 1.0292 & 1.0000 \\
\hline \multirow{3}{*}{4} & 2 & 0.9947 & 1.0445 & 1.2316 & 0.9947 & 1.0066 & 1.0238 & 0.9947 \\
\hline & 3 & 0.9882 & 1.0376 & 1.2235 & 0.9882 & 1.0000 & 1.0171 & 0.9882 \\
\hline & 4 & 0.9947 & 1.0445 & 1.2316 & 0.9947 & 1.0066 & 1.0238 & 0.9947 \\
\hline \multirow{4}{*}{5} & 2 & 0.9739 & 1.0226 & 1.2058 & 0.9739 & 0.9855 & 1.0023 & 0.9739 \\
\hline & 3 & 0.9882 & 1.0376 & 1.2235 & 0.9882 & 1.0000 & 1.0171 & 0.9882 \\
\hline & 4 & 0.9882 & 1.0376 & 1.2235 & 0.9882 & 1.0000 & 1.0171 & 0.9882 \\
\hline & 5 & 0.9739 & 1.0226 & 1.2058 & 0.9739 & 0.9855 & 1.0023 & 0.9739 \\
\hline \multirow{5}{*}{6} & 2 & 0.9391 & 0.9861 & 1.1627 & 0.9391 & 0.9503 & 0.9665 & 0.9391 \\
\hline & 3 & 0.9958 & 1.0455 & 1.2328 & 0.9958 & 1.0076 & 1.0248 & 0.9958 \\
\hline & 4 & 0.9716 & 1.0202 & 1.2030 & 0.9716 & 0.9832 & 1.0000 & 0.9716 \\
\hline & 5 & 0.9958 & 1.0455 & 1.2328 & 0.9958 & 1.0076 & 1.0248 & 0.9958 \\
\hline & 6 & 0.9391 & 0.9861 & 1.1627 & 0.9391 & 0.9503 & 0.9665 & 0.9391 \\
\hline
\end{tabular}

Table 5. AREs of $T_{c, d i ; i, j}$ for Laplace distribution w.r.t. different tests

\begin{tabular}{|c|c|c|c|c|c|c|c|c|}
\hline & & \multicolumn{7}{|c|}{ Test } \\
\hline$c+d$ & $i+j$ & $\begin{array}{c}M W \\
(k=3)\end{array}$ & $\begin{array}{c}M W \\
(k=5)\end{array}$ & $\begin{array}{c}M W \\
(k=7)\end{array}$ & $\begin{array}{c}P_{1,1} \\
(\text { any } k)\end{array}$ & $\begin{array}{c}P_{1,3} \\
(\text { any } k)\end{array}$ & $\begin{array}{c}P_{3,3} \\
(\text { any } k)\end{array}$ & $\begin{array}{c}B \\
(\text { any } k)\end{array}$ \\
\hline 2 & 2 & 1.0000 & 1.0500 & 1.2381 & 1.0000 & 0.9326 & 0.8999 & 1.0000 \\
\hline \multirow{2}{*}{3} & 2 & 1.0000 & 1.0500 & 1.2381 & 1.0000 & 0.9326 & 0.8999 & 1.0000 \\
\hline & 3 & 1.0000 & 1.0500 & 1.2381 & 1.0000 & 0.9326 & 0.8999 & 1.0000 \\
\hline \multirow{3}{*}{4} & 2 & 0.9402 & 0.9873 & 1.1641 & 0.9402 & 0.8768 & 0.8461 & 0.9402 \\
\hline & 3 & 1.0723 & 1.1259 & 1.3276 & 1.0723 & 1.0000 & 0.9649 & 1.0723 \\
\hline & 4 & 0.9402 & 0.9873 & 1.1641 & 0.9402 & 0.8768 & 0.8461 & 0.9402 \\
\hline \multirow{4}{*}{5} & 2 & 0.8560 & 0.8988 & 1.0598 & 0.8560 & 0.7983 & 0.7703 & 0.8560 \\
\hline & 3 & 1.0723 & 1.1259 & 1.3276 & 1.0723 & 1.0000 & 0.9649 & 1.0723 \\
\hline & 4 & 1.0723 & 1.1259 & 1.3276 & 1.0723 & 1.0000 & 0.9649 & 1.0723 \\
\hline & 5 & 0.8560 & 0.8988 & 1.0598 & 0.8560 & 0.7983 & 0.7703 & 0.8560 \\
\hline \multirow{5}{*}{6} & 2 & 0.7677 & 0.8061 & 0.9505 & 0.7677 & 0.7160 & 0.6909 & 0.7677 \\
\hline & 3 & 1.0377 & 1.0896 & 1.2847 & 1.0377 & 0.9677 & 0.9338 & 1.0377 \\
\hline & 4 & 1.1113 & 1.1668 & 1.3759 & 1.1113 & 1.0364 & 1.0000 & 1.1113 \\
\hline & 5 & 1.0377 & 1.0896 & 1.2847 & 1.0377 & 0.9677 & 0.9338 & 1.0377 \\
\hline & 6 & 0.7677 & 0.8061 & 0.9505 & 0.7677 & 0.7160 & 0.6909 & 0.7677 \\
\hline
\end{tabular}


International Journal of Mathematical, Engineering and Management Sciences

Vol. 3, No. 4, 498-512, 2018

https://dx.doi.org/10.33889/IJMEMS.2018.3.4-036

Table 6. AREs of $T_{c, d ; i, j}$ for Cauchy distribution w.r.t. different tests

\begin{tabular}{|c|c|c|c|c|c|c|c|c|}
\hline & & \multicolumn{7}{|c|}{ Test } \\
\hline \multirow{3}{*}{$c+d$} & $i+j$ & $\begin{array}{c}M W \\
(k=3)\end{array}$ & $\begin{array}{c}M W \\
(k=5)\end{array}$ & $\begin{array}{c}M W \\
(k=7)\end{array}$ & $\begin{array}{c}P_{1,1} \\
\text { (any } k)\end{array}$ & $\begin{array}{c}P_{1,3} \\
(\text { any } k)\end{array}$ & $\begin{array}{c}P_{3,3} \\
(\text { any } k)\end{array}$ & $\begin{array}{c}B \\
\text { (any } k)\end{array}$ \\
\hline 2 & 2 & 1.0000 & 1.0500 & 1.2381 & 1.0000 & 0.8570 & 0.7960 & 1.0000 \\
\hline \multirow{3}{*}{3} & 2 & 1.0000 & 1.0500 & 1.2381 & 1.0000 & 0.8570 & 0.7960 & 1.0000 \\
\cline { 2 - 9 } & 3 & 1.0000 & 1.0500 & 1.2381 & 1.0000 & 0.8570 & 0.7960 & 1.0000 \\
\hline \multirow{4}{*}{4} & 2 & 0.8831 & 0.9273 & 1.0934 & 0.8831 & 0.7568 & 0.7030 & 0.8831 \\
\cline { 2 - 10 } & 3 & 1.1669 & 1.2252 & 1.4447 & 1.1669 & 1.0000 & 0.9288 & 1.1669 \\
\cline { 2 - 10 } & 4 & 0.8831 & 0.9273 & 1.0934 & 0.8831 & 0.7568 & 0.7030 & 0.8831 \\
\hline \multirow{5}{*}{5} & 2 & 0.7372 & 0.7741 & 0.9128 & 0.7372 & 0.6318 & 0.5868 & 0.7372 \\
\cline { 2 - 10 } & 3 & 1.1669 & 1.2252 & 1.4447 & 1.1669 & 1.0000 & 0.9288 & 1.1669 \\
\cline { 2 - 10 } & 4 & 1.1669 & 1.2252 & 1.4447 & 1.1669 & 1.0000 & 0.9288 & 1.1669 \\
\cline { 2 - 10 } & 5 & 0.7372 & 0.7741 & 0.9128 & 0.7372 & 0.6318 & 0.5868 & 0.7372 \\
\hline \multirow{5}{*}{6} & 2 & 0.5988 & 0.6288 & 0.7414 & 0.5988 & 0.5132 & 0.4767 & 0.5988 \\
\cline { 2 - 9 } & 3 & 1.0953 & 1.1501 & 1.3561 & 1.0953 & 0.9387 & 0.8718 & 1.0953 \\
\cline { 2 - 9 } & 4 & 1.2563 & 1.3191 & 1.5554 & 1.2563 & 1.0766 & 1.0000 & 1.2563 \\
\cline { 2 - 9 } & 5 & 1.0953 & 1.1501 & 1.3561 & 1.0953 & 0.9387 & 0.8718 & 1.0953 \\
\cline { 2 - 9 } & 6 & 0.5988 & 0.6288 & 0.7414 & 0.5988 & 0.5132 & 0.4767 & 0.5988 \\
\hline
\end{tabular}

Table 7. AREs of $T_{c, d ; i, j}$ for Gumbel distribution w.r.t. different tests

\begin{tabular}{|c|c|c|c|c|c|c|c|c|}
\hline & & \multicolumn{7}{|c|}{ Test } \\
\hline$c+d$ & $i+j$ & $\begin{array}{c}M W \\
(k=3)\end{array}$ & $\begin{array}{c}M W \\
(k=5)\end{array}$ & $\begin{array}{c}M W \\
(k=7)\end{array}$ & $\begin{array}{c}P_{1,1} \\
(\text { any } k)\end{array}$ & $\begin{array}{c}P_{1,3} \\
(\text { any } k)\end{array}$ & $\begin{array}{c}P_{3,3} \\
(\text { any } k)\end{array}$ & $\begin{array}{c}B \\
(\text { any } k)\end{array}$ \\
\hline 2 & 2 & 1.0000 & 1.0500 & 1.2381 & 1.0000 & 1.0706 & 1.1185 & 1.0000 \\
\hline \multirow{2}{*}{3} & 2 & 1.0000 & 1.0500 & 1.2381 & 1.0000 & 1.0706 & 1.1185 & 1.0000 \\
\hline & 3 & 1.0000 & 1.0500 & 1.2381 & 1.0000 & 1.0706 & 1.1185 & 1.0000 \\
\hline \multirow{3}{*}{4} & 2 & 1.0319 & 1.0835 & 1.2776 & 1.0319 & 1.1047 & 1.1542 & 1.0319 \\
\hline & 3 & 0.9341 & 0.9808 & 1.1565 & 0.9341 & 1.0000 & 1.0447 & 0.9341 \\
\hline & 4 & 1.0319 & 1.0835 & 1.2776 & 1.0319 & 1.1047 & 1.1542 & 1.0319 \\
\hline \multirow{4}{*}{5} & 2 & 1.0568 & 1.1096 & 1.3084 & 1.0568 & 1.1313 & 1.1819 & 1.0568 \\
\hline & 3 & 0.9341 & 0.9808 & 1.1565 & 0.9341 & 1.0000 & 1.0447 & 0.9341 \\
\hline & 4 & 0.9341 & 0.9808 & 1.1565 & 0.9341 & 1.0000 & 1.0447 & 0.9341 \\
\hline & 5 & 1.0568 & 1.1096 & 1.3084 & 1.0568 & 1.1313 & 1.1819 & 1.0568 \\
\hline \multirow{5}{*}{6} & 2 & 1.0663 & 1.1196 & 1.3201 & 1.0663 & 1.1415 & 1.1926 & 1.0663 \\
\hline & 3 & 0.9601 & 1.0081 & 1.1887 & 0.9601 & 1.0279 & 1.0739 & 0.9601 \\
\hline & 4 & 0.8941 & 0.9388 & 1.1070 & 0.8941 & 0.9572 & 1.0000 & 0.8941 \\
\hline & 5 & 0.9601 & 1.0081 & 1.1887 & 0.9601 & 1.0279 & 1.0739 & 0.9601 \\
\hline & 6 & 1.0663 & 1.1196 & 1.3201 & 1.0663 & 1.1415 & 1.1926 & 1.0663 \\
\hline
\end{tabular}


International Journal of Mathematical, Engineering and Management Sciences

Vol. 3, No. 4, 498-512, 2018

https://dx.doi.org/10.33889/IJMEMS.2018.3.4-036

From the tables of AREs, we observed that

$>$ Performance of $T_{c, d ; i, j}$ tests depends upon the tail behaviour of the underlying distribution.

$>$ For Light tailed or skewed distributions, one should choose $(c, d ; i, j)$ in such a way that $(c+$ d) as maximum as possible with $(i+j)$ is either equal to 2 or equal to $(c+d)$, to have maximum efficiency.

$>$ For Medium tailed distributions, one should choose $(c, d ; i, j)$ in such a way that $(c+d)=5$ with $(i+j)$ is either equal to 2 or equal to 5 , to have maximum efficiency. In case of Logistic distribution, $T_{c, d ; i, j}$ test with $(c+d)=(i+j)=2$ has maximum efficiency.

For heavy tailed distributions, choose $(c, d ; i, j)$ in such a way that $(c+d)$ as maximum as possible with $(i+j)$ is greatest integer less than or equal to $(c+d+2) / 2$, to have maximum efficiency.

\section{An Illustrative Example}

To see the execution of the proposed class of tests, we consider the data obtained on Wechsler Adult Intelligence Scale (WAIS) by R. D. Norman. The data is given by Hollander et al. (2013, p.242). It is commonly believed that learning ability is increasing function of age up to a certain point and then it starts decreasing with further increasing of age. The data considered here are the WAIS values of the males of five age groups as $16-19,20-34,35-54,55-69$, and $\geq 70$. Now, we wish to test whether the WAIS values of all the age groups are same against the alternative that WAIS values are increasing up to age group $35-54$ and then start decreasing with further increasing of age.

By using Kolmogorov-Smirnov test, the given data set follows Normal distribution at 5\% level of significance. So, by using observations from Section 5 of AREs of $T_{c, d ; i, j}$ tests, one should choose $(c, d ; i, j)$ in such a way that $(c+d)=5$ with $(i+j)$ is either equal to 2 or equal to 5 .

The computed value of $T_{c, d ; i, j}$, with $(c, d ; i, j)=(3,2 ; 1,1)$ or $(3,2 ; 3,2)$ is equal to 1.08 and corresponding $p$-value is 0.0000632 . Therefore, test is to reject $H_{0}$ at $5 \%$ level of significance. The computed value of Mack and Wolfe (1981) test statistics for same data is 6.22 and corresponding $p$-value is 0.00325 . Both tests are rejecting $H_{0}$ at $5 \%$ level of significance but $T_{c, d ; i, j}$ test with $(c, d ; i, j)=(3,2 ; 1,1)$ or $(3,2 ; 3,2)$ clarifies it more significantly.

As test is to reject $H_{0}$, we can say that learning ability is increasing function of age up to age group $35-54$ and then start decreasing with further increasing of age.

\section{Simulation Study}

To assess the power of the proposed $T_{c, d ; i, j}$ and $V_{c, d ; i, j}$ tests, Monte Carlo simulation study is carried out. We consider three independent random samples i.e. $k=3$ with umbrella peak at $2^{\text {nd }}$ sample i.e. $p=2$. Data is generated from Normal distribution and sample size $n=10$ (10) 30 with shift parameter $\theta=\theta_{2}-\theta_{1}=\theta_{2}-\theta_{3}=0.3(0.3) 0.9$. The computation of power is based on 10,000 repetitions and level of significance is fixed at 0.05 . The estimated power is given in Tables 8-9. 
International Journal of Mathematical, Engineering and Management Sciences

Vol. 3, No. 4, 498-512, 2018

https://dx.doi.org/10.33889/IJMEMS.2018.3.4-036

Table 8. Estimated power of $T_{c, d ; i, j}$ for Normal distribution

\begin{tabular}{|c|c|c|c|c|c|c|c|c|c|}
\hline & & \multicolumn{9}{|c|}{$(c+d ; i+j)$} \\
\hline$n$ & $\theta$ & $(2 ; 2)$ & $(3 ; 2)$ & $(4 ; 2)$ & $(5 ; 2)$ & $(5 ; 3)$ & $(6 ; 2)$ & $(6 ; 3)$ & $(6 ; 4)$ \\
\hline \multirow{4}{*}{10} & 0.3 & 0.1959 & 0.1978 & 0.1989 & 0.2024 & 0.1899 & 0.2008 & 0.1900 & 0.1808 \\
\cline { 2 - 10 } & 0.6 & 0.4479 & 0.4489 & 0.4513 & 0.4540 & 0.4272 & 0.4526 & 0.4288 & 0.4267 \\
\cline { 2 - 10 } & 0.9 & 0.7207 & 0.7279 & 0.7294 & 0.7346 & 0.7031 & 0.7302 & 0.7078 & 0.6908 \\
\hline \multirow{4}{*}{20} & 0.3 & 0.2778 & 0.2795 & 0.2829 & 0.2870 & 0.2586 & 0.2855 & 0.2597 & 0.2441 \\
\cline { 2 - 10 } & 0.6 & 0.6832 & 0.6834 & 0.7034 & 0.7102 & 0.6650 & 0.7061 & 0.6682 & 0.6560 \\
\cline { 2 - 10 } & 0.9 & 0.9389 & 0.9391 & 0.9413 & 0.9508 & 0.9184 & 0.9459 & 0.9206 & 0.9118 \\
\hline \multirow{4}{*}{30} & 0.3 & 0.3679 & 0.3684 & 0.3698 & 0.3756 & 0.3480 & 0.3723 & 0.3489 & 0.3373 \\
\cline { 2 - 10 } & 0.6 & 0.8319 & 0.8434 & 0.8481 & 0.8564 & 0.8129 & 0.8516 & 0.8150 & 0.8029 \\
\cline { 2 - 10 } & 0.9 & 0.9853 & 0.9861 & 0.9880 & 0.9935 & 0.9664 & 0.9902 & 0.9673 & 0.9568 \\
\hline
\end{tabular}

Table 9. Estimated power of $V_{c, d ; i, j}$ for Normal distribution

\begin{tabular}{|c|c|c|c|c|c|c|c|c|c|}
\hline & & \multicolumn{7}{|c|}{$(c+d ; i+j)$} \\
\hline$n$ & $\theta$ & $(2 ; 2)$ & $(3 ; 2)$ & $(4 ; 2)$ & $(5 ; 2)$ & $(5 ; 3)$ & $(6 ; 2)$ & $(6 ; 3)$ & $(6 ; 4)$ \\
\hline \multirow{3}{*}{1} & 0.3 & 0.1948 & 0.1951 & 0.1986 & 0.2013 & 0.1871 & 0.1997 & 0.1847 & 0.1795 \\
\cline { 2 - 10 } 0 & 0.6 & 0.4411 & 0.4478 & 0.4497 & 0.4531 & 0.4226 & 0.4513 & 0.4195 & 0.4188 \\
\cline { 2 - 10 } & 0.9 & 0.7213 & 0.7252 & 0.7285 & 0.7341 & 0.7002 & 0.7291 & 0.6973 & 0.6817 \\
\hline \multirow{3}{*}{2} & 0.3 & 0.2698 & 0.2758 & 0.2827 & 0.2864 & 0.2584 & 0.2854 & 0.2891 & 0.2430 \\
\cline { 2 - 10 } 0 & 0.6 & 0.6764 & 0.6830 & 0.6927 & 0.7098 & 0.6643 & 0.7057 & 0.6678 & 0.6542 \\
\cline { 2 - 10 } & 0.9 & 0.9368 & 0.9373 & 0.9392 & 0.9503 & 0.9172 & 0.9448 & 0.9194 & 0.9102 \\
\hline \multirow{3}{*}{3} & 0.3 & 0.3590 & 0.3610 & 0.3694 & 0.3748 & 0.3480 & 0.3718 & 0.3485 & 0.3365 \\
\cline { 2 - 9 } 0 & 0.6 & 0.8211 & 0.8290 & 0.8475 & 0.8560 & 0.8117 & 0.8504 & 0.8139 & 0.7986 \\
\cline { 2 - 9 } & 0.9 & 0.9842 & 0.9858 & 0.9876 & 0.9932 & 0.9659 & 0.9893 & 0.9669 & 0.9541 \\
\hline
\end{tabular}

Based on the power computations, we observed that for proposed tests $T_{c, d ; i, j}$ and $V_{c, d ; i, j}$, shift of order 0.9 is detected at sample size $n=20$ for $(c, d ; i, j)$ in such a way that $(c+d)=5$ with $(i+j)=2$ and required more sample size for other combinations of $(c, d ; i, j)$ to detect the shift of same order. This authenticates the computations of AREs as well.

\section{Acknowledgment}

The authors thank anonymous reviewers for their valuable suggestions, which led to an improvement of the paper.

\section{References}

Amita, \& Kochar, S. C. (1989). Some distribution free tests for testing homogeneity of location parameters against ordered alternatives. Journal of Indian Statistical Association, 27, 1-8.

Archambault, W. A. T., Mack, G. A., \& Wolfe, D. A. (1977). K-sample rank tests using pair-specific scoring functions. Canadian Journal of Statistics, 5(2), 195-207. 
International Journal of Mathematical, Engineering and Management Sciences

Vol. 3, No. 4, 498-512, 2018

https://dx.doi.org/10.33889/IJMEMS.2018.3.4-036

Bhat, S. V. (2009). Simple K-sample rank tests for umbrella alternatives. Research Journal of Mathematics and Statistics, 1(1), 27-29.

Büning, H., \& Kössler, W. (1996). Robustness and efficiency of some tests for ordered alternatives in the csample location problem. Journal of Statistical Computation and Simulation, 55(4), 337-352.

Büning, H., \& Kössler, W. (1997). Power of Some Tests for Umbrella Alternatives in the Multi-Sample Location Problem. Biometrical Journal, 39(4), 481-494.

Deshpande, J. V., \& Kochar, S. C. (1982). Some competitors of Wilcoxon-Mann-Whitney test for location alternatives. Journal of Indian Statistical Association, 19, 9-18.

Gökpinar, E., \& Gökpinar, F. (2016). A modified Mack-Wolfe test for the umbrella alternative problem. Communications in Statistics-Theory and Methods, 45(24), 7226-7241.

Goyal, M., \& Kumar, N. (2017). A general class of tests for testing homogeneity of location parameters against ordered alternatives. Proceedings in Mathematics and Statistics, under the title Logistics, Supply Chain and Financial Predictive Analytics: Theory and Practices, Springer (accepted).

Hollander, M., Wolfe, D. A., \& Chicken, E. (2013). Nonparametric statistical methods (Vol. 751). John Wiley \& Sons.

Jonckheere, A. R. (1954). A distribution-free $k$-samples test against ordered alternatives. Biomerika, $41(1 / 2), 133-145$.

Kössler, W. (2005). Some c-sample rank tests of homogeneity against ordered alternatives based on Ustatistics. Journal of Nonparametric Statistics, 17(7), 777-795.

Kössler, W. (2006). Some c-sample rank tests of homogeneity against umbrella alternatives with unknown peak. Journal of Statistical Computation and Simulation, 76(1), 57-74.

Kumar, N. (1997). A class of two-sample tests for location based on sub-sample medians. Communication in Statistics - Theory and Methods, 26(4), 943-951.

Kumar, N. (2015). A class of nonparametric tests based on sample quantiles. Journal of Combinatorics, Information \& System Sciences, 40(1-4), 211-223.

Kumar, N., \& Goyal, M. (2018). Jonckheere type test procedure with optimal criterion under order restrictions. American Journal of Mathematical and Management Sciences. doi: https://doi.org/10.1080/01966324.2017.1404948.

Kumar, N., Gill, A. N., \& Dhawan, A. K. (1994b). A class of distribution-free statistics for homogeneity against ordered alternatives. South African Statistical Journal, 28(1), 55-65.

Kumar, N., Gill, A. N., \& Mehta, G. P. (1994a). Distribution-free test for homogeneity against ordered alternatives. Communication in Statistics - Theory and Methods, 23(4), 1247-1256.

Kumar, N., Singh, R. S., \& Öztürk, Ö. (2003). A new class of distribution-free tests for location parameters. Sequential Analysis, 22(1-2), 107-128.

Lehmann, E. L. (1963). Robust estimation in analysis of variance. Annals of Mathematical Statistics, 34(3), 957-966.

Mack, G. A., \& Wolfe, D. A. (1981). K-sample rank tests for umbrella alternatives. Journal of the American Statistical Association, 76(373), 175-181.

Mann, H. B., \& Whitney, D. R. (1947). On a test of whether one of the random variables is stochastically greater than the other. Annals of Mathematical Statistics, 18(1), 50-60.

Patil, A. B. (2007). A Study on Distribution-Free Test Statistics. Thesis submitted to the Karnataka University, Dharwad. 
International Journal of Mathematical, Engineering and Management Sciences

Vol. 3, No. 4, 498-512, 2018

https://dx.doi.org/10.33889/IJMEMS.2018.3.4-036

Puri, M. L. (1965). Some distribution-free k-sample rank tests of homogeneity against ordered alternatives. Communications on Pure Applied Mathematics, 18(1-2), 51-63.

Rao, C. R. (1973). Linear Statistical Inference and its Applications. Wiley Eastern Ltd., New York.

Serfling, R. J. (1980). Approximation Theorems of Mathematical Statistics. John Wiley and Sons, New York.

Simpson, D. G., \& Margolin, B. H. (1986). Recursive nonparametric testing for dose-response relationships subject to downturns at high doses. Biometrika, 73(3), 589-596.

Terpstra, T. J. (1952). The asymptotic normality and consistency of Kendall's test against trend, when ties are present in one ranking. Indigationes Mathematicae, 14(3), 327-333.

Wilcoxon, F. (1945). Individual comparisons by rank methods. Biometrics Bulletin, 1(6), 80-83. 